\title{
Phase transitions in a complex network
}

\author{
Charles Radin and Lorenzo Sadun \\ Department of Mathematics, The University of Texas at Austin, Austin, TX 78712, USA \\ E-mail: radin@math.utexas.edu and sadun@math.utexas.edu
}

Received 1 March 2013, in final form 6 June 2013

Published 10 July 2013

Online at stacks.iop.org/JPhysA/46/305002

\begin{abstract}
We study a mean field model of a complex network, focusing on edge and triangle densities. Our first result is the derivation of a variational characterization of the entropy density, compatible with the infinite node limit. We then determine the optimizing graphs for small triangle density and a range of edge density, though we can only prove they are local, not global, maxima of the entropy density. With this assumption we then prove that the resulting entropy density must lose its analyticity in various regimes. In particular this implies the existence of a phase transition between distinct heterogeneous multipartite phases at low triangle density, and a phase transition between these phases and the disordered phase at high triangle density.
\end{abstract}

PACS numbers: 89.75.Fb, 05.70.Fh

Mathematics Subject Classification: 82B26, 05C35, 05C80

\section{Introduction}

Exponential random graph models are a well known class of complex networks; see [Ne] and references therein. Using the language of statistical mechanics they are mean field models, in the grand canonical ensemble, with a variety of possible many-body interactions appropriate to the model's use. Phase transitions, which require an infinite node limit, have been proven for them [CD, RY] using the recently developed 'graphon' formalism [Lov] in place of the infinite volume limit formalism [R1, R2] used in statistical mechanics. Exponential random graph models are mean field models and therefore the analogues of the various statistical mechanics ensembles (microcanonical, grand canonical, pressure, ...) which are equivalent in the infinite volume limit for particle systems with short range interactions [R1], need not be equivalent in these mean field models; see for instance [TET]. (Equivalence of ensembles is discussed further in the conclusion.) In this work we use the microcanonical ensemble of one of the best known exponential random graph models, one originally formulated by Strauss [St], and give evidence of phase transitions which are not as accessible in the grand canonical ensemble. The transitions previously analyzed for a wide class of exponential random graphs are similar to liquid/gas transitions in that they are transitions between graphs of similar character, of the 


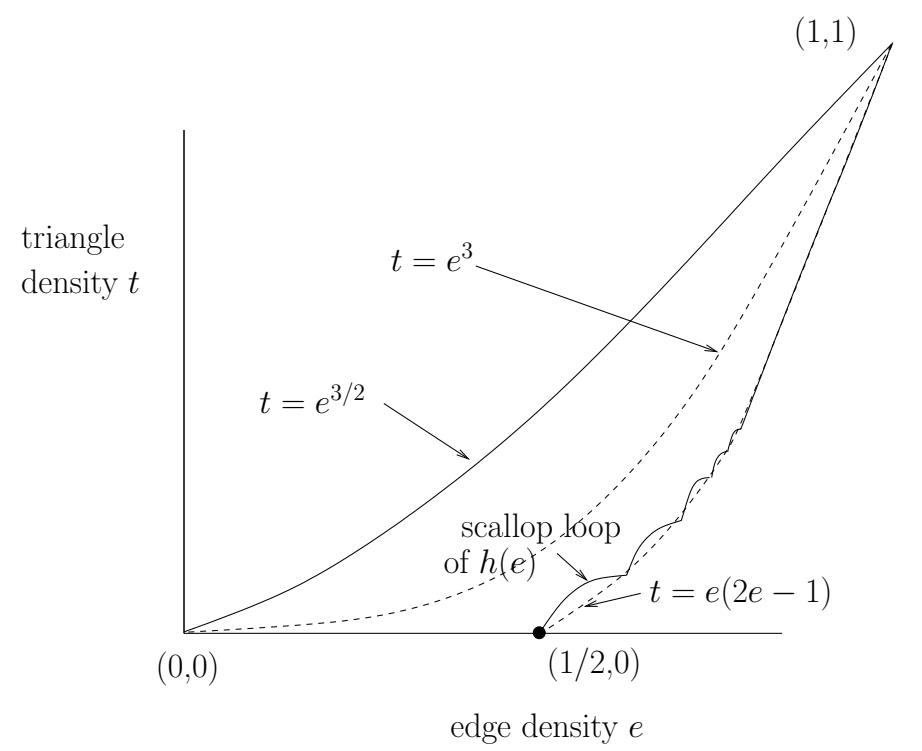

Figure 1. The microcanonical phase space $R$, outlined in solid lines.

same (fluid-like) phase [RY], while the transitions we focus on in the microcanonical ensemble are analogous to solid/solid transitions, transitions between graphs of different phases. (See [AR] for a more primitive grand canonical analysis of these phases.)

We need some network notation. Consider the set $\hat{G}^{n}$ of simple graphs $G$ with set $V(G)$ of (labeled) vertices, edge set $E(G)$ and triangle set $T(G)$, where the cardinality $|V(G)|=n$. ('Simple' means the edges are undirected and there are no multiple edges or loops.) Think of an unordered pair of vertices as a point in an abstract space, an edge as a particle that may occupy that point, and a triangle as a many-body interaction energy associated with its edges, so the microcanonical partition function, $Z_{e, t}^{n, \delta}$, is the number of simple graphs such that:

$e(G) \equiv \frac{|E(G)|}{\left(\begin{array}{l}n \\ 2\end{array}\right)} \in(e-\delta, e+\delta) \quad$ and $\quad t(G) \equiv \frac{|T(G)|}{\left(\begin{array}{l}n \\ 3\end{array}\right)} \in(t-\delta, t+\delta)$.

Graphs in $\cup_{n \geqslant 1} \hat{G}^{n}$ are known to have edge and triangle densities, $(e, t)$, dense in the region $R$ in the $e, t$-plane bounded by three curves, $c_{1}:\left(e, e^{3 / 2}\right), \quad 0 \leqslant e \leqslant 1$, the line $l_{1}:(e, 0), \quad 0 \leqslant e \leqslant 1 / 2$ and a certain scalloped curve $(e, h(e)), 1 / 2 \leqslant e \leqslant 1$, lying above the curve $\left(e, e(2 e-1), 1 / 2 \leqslant e \leqslant 1\right.$, and meeting it when $e=e_{k}=k /(k+1), k \geqslant 1$; see $[\mathrm{PR}]$ and references therein, and figure 1.

We are interested in the relative density of graphs in $R$, more precisely in the entropy, the exponential rate of growth of the number of graphs as $n$ grows, as follows. First consider

$$
s_{e, t}^{n, \delta}=\frac{\ln \left(Z_{e, t}^{n, \delta}\right)}{n^{2}}, \text { and then } s_{e, t}=\lim _{\delta \rightarrow 0^{+}} \lim _{n \rightarrow \infty} s_{e, t}^{n, \delta} .
$$

(The existence of the double limit will be proven.) We will measure the growth rate by the entropy density $s_{e, t}$, and the main question of interest for us is the existence of phase transitions (i.e. lack of analyticity of $s_{e, t}$ ) near the lower boundary of $R$ in figure 1 . The lower boundary consists of the scalloped curve together with the 'first scallop', the line from $(0,0)$ to $(1 / 2,0)$.

We now need to review some notation and results concerning graphons, as recently developed in [LS1, LS2, BCLSV, BCL, LS3]. See also the recent book [Lov]. 


\section{Graphons}

Consider the set $\mathcal{W}$ of all symmetric, measurable functions

$$
g:(x, y) \in[0,1]^{2} \rightarrow g(x, y) \in[0,1] .
$$

Think of each axis as a continuous set of vertices of a graph. For a graph $G \in \hat{G}^{n}$ one associates

$$
g^{G}(x, y)= \begin{cases}1 & \text { if }(\lceil n x\rceil,\lceil n y\rceil) \text { is an edge of } G \\ 0 & \text { otherwise, }\end{cases}
$$

where $\lceil y\rceil$ denotes the smallest integer greater than or equal to $y$. For $g \in \mathcal{W}$ and simple graph $H$ we define

$$
t(H, g) \equiv \int_{[0,1]^{\ell}} \prod_{(i, j) \in E(H)} g\left(x_{i}, x_{j}\right) \mathrm{d} x_{1} \cdots \mathrm{d} x_{\ell},
$$

where $\ell=|V(H)|$, and note that for a graph $G, t\left(H, g^{G}\right)$ is the density of graph homomorphisms $H \rightarrow G$ :

$$
\frac{|\operatorname{hom}(H, G)|}{|V(G)|^{|V(H)|}} .
$$

We define an equivalence relation on $\mathcal{W}$ as follows: $f \sim g$ if and only if $t(H, f)=t(H, g)$ for every simple graph $H$. Elements of $\mathcal{W}$ are called 'graphons', elements of the quotient space $\tilde{\mathcal{W}}$ are called 'reduced graphons', and the class containing $g \in \mathcal{W}$ is denoted $\tilde{g}$. Equivalent functions in $\mathcal{W}$ differ by a change of variables in the following sense. Let $\Sigma$ be the space of measure preserving maps $\sigma:[0,1] \rightarrow[0,1]$, and for $f$ in $\mathcal{W}$ and $\sigma \in \Sigma$, let $f_{\sigma}(x, y) \equiv f(\sigma(x), \sigma(y))$. Then $f \sim g$ if and only if there exist $\sigma, \sigma^{\prime}$ in $\Sigma$ such that $f_{\sigma}=g_{\sigma^{\prime}}$ almost everywhere; see corollary 2.2 in [BCL]. The space $\mathcal{W}$ is compact with respect to the 'cut metric' defined as follows. First, on $\mathcal{W}$ define:

$$
d_{\mathbf{.}}(f, g) \equiv \sup _{S, T \subseteq[0,1]}\left|\int_{S \times T}[f(x, y)-g(x, y)] \mathrm{d} x \mathrm{~d} y\right| .
$$

Then on $\tilde{\mathcal{W}}$ define the cut metric by:

$$
\tilde{d}_{\mathbf{\bullet}}(\tilde{f}, \tilde{g}) \equiv \inf _{\sigma, \sigma^{\prime} \in \Sigma} d_{\mathbf{\bullet}}\left(f_{\sigma}, g_{\sigma^{\prime}}\right) .
$$

We will use the fact, which follows easily from lemma 4.1 in [LS1], that the cut metric is equivalent to the metric

$$
\delta_{\text {hom }}(\tilde{f}, \tilde{g}) \equiv \sum_{j \geqslant 1} \frac{1}{2^{j}}\left|t\left(H_{j}, f\right)-t\left(H_{j}, g\right)\right|,
$$

where $\left\{H_{j}\right\}$ is a countable set of simple graphs, one from each graph-equivalence class. Also note that if each vertex of a finite graph is split into the same number of 'twins', each connected to the same vertices, the result stays in the same equivalence class, so for a convergent sequence $\tilde{g}^{G_{j}}$ one may assume $\left|V\left(G_{j}\right)\right| \rightarrow \infty$.

The value of this graphon formalism here is that one can use large deviations on graphs with independent edges, proven in $[\mathrm{CV}]$, to give an optimization formula for $s_{e, t}$, which allows us to analyze $s_{e, t}$ near the graphons of minimal triangle density, the lower boundary of $R$ in figure 1. We next use the large deviations rate function for graphs with independent edges to give a variational characterization for the entropy density. (There is a variational characterization in [CD] of the free energy density in the grand canonical ensemble.) 


\section{A variational characterization of the entropy density}

Theorem 3.1. For any possible pair $(e, t), s_{e, t}=-\min I(g)$, where the minimum is over all graphons $g$ with $e(g)=e$ and $t(g)=t$, where

$e(g)=\int_{0}^{1} \int_{0}^{1} g(x, y) \mathrm{d} x \mathrm{~d} y, \quad t(g)=\int_{0}^{1} \int_{0}^{1} \int_{0}^{1} g(x, y) g(y, z) g(z, x) \mathrm{d} x \mathrm{~d} y \mathrm{~d} z$,

and the rate function is

$I(g)=\int_{0}^{1} \int_{0}^{1} I_{0}(g(x, y)) \mathrm{d} x \mathrm{~d} y$, where $I_{0}(u)=\frac{1}{2}[u \ln (u)+(1-u) \ln (1-u)]$.

Proof. We first prove that $s_{e, t}$ is well-defined. A priori all we know is that $\lim \inf \ln \left(Z_{e, t}^{n, \delta}\right) / n^{2}$ and $\lim \sup \ln \left(Z_{e, t}^{n, \delta}\right) / n^{2}$ exist as $n \rightarrow \infty$. However, we will show that they both approach $-\min I(g)$ as $\delta \rightarrow 0^{+}$.

We need to define a few sets. Let $U_{\delta}$ be the set of graphons $g$ with $e(g)$ and $t(g)$ strictly within $\delta$ of $e$ and $t$, i.e. the preimage of an open square of side $2 \delta$ in $(e, t)$-space, and let $F_{\delta}$ be the preimage of the closed square. Let $\tilde{U}_{\delta}$ and $\tilde{F}_{\delta}$ be the corresponding sets in $\tilde{\mathcal{W}}$. Let $\left|U_{\delta}^{n}\right|$ and $\left|F_{\delta}^{n}\right|$ denote the number of graphs with $n$ vertices whose checkerboard graphons (4) lie in $U_{\delta}$ or $F_{\delta}$. The large deviation principle, theorem 2.3 of [CV], implies that:

$$
\limsup _{n \rightarrow \infty} \frac{\ln \left|F_{\delta}^{n}\right|}{n^{2}} \leqslant-\inf _{\tilde{g} \in \tilde{F}_{\delta}} I(\tilde{g})
$$

which also equals $-\inf _{g \in F_{\delta}} I(g)$, and that

$$
\liminf _{n \rightarrow \infty} \frac{\ln \left|U_{\delta}^{n}\right|}{n^{2}} \geqslant-\inf _{\tilde{g} \in \tilde{U}_{\delta}} I(\tilde{g})
$$

which also equals $-\inf _{g \in U_{\delta}} I(g)$. This yields a chain of inequalities

$$
-\inf _{U_{\delta}} I(g) \leqslant \liminf \frac{\ln \left|U_{\delta}^{n}\right|}{n^{2}} \leqslant \limsup \frac{\ln \left|U_{\delta}^{n}\right|}{n^{2}} \leqslant \limsup \frac{\ln \left|F_{\delta}^{n}\right|}{n^{2}} \leqslant-\inf _{F_{\delta}} I(g) \leqslant-\inf _{U_{\delta+\delta^{2}}} I(g) .
$$

As $\delta \rightarrow 0^{+}$, the limits of $-\inf _{U_{\delta}} I(g)$ and $-\inf _{U_{\delta+\delta^{2}}} I(g)$ are the same, and everything in between is trapped.

So far we have proven that

$$
s_{e, t}=-\lim _{\delta \rightarrow 0^{+}} \inf _{\delta} I(g) .
$$

Next we must show that the right hand side is equal to $-\min _{F_{0}} I(g)$. By definition, we can find a sequence of reduced graphons $\tilde{g}_{\delta} \in \tilde{U}_{\delta}$ such that $\lim _{\delta \rightarrow 0} I\left(\tilde{g}_{\delta}\right)=\liminf _{U_{\delta}} I(g)$. Since $\tilde{W}$ is compact, these reduced graphons converge to a reduced graphon $\tilde{g}_{0}$, represented by a graphon $g_{0} \in F_{0}$. Since $I$ is lower-semicontinuous [CV], $I\left(g_{0}\right) \leqslant \lim I\left(g_{\delta}\right)$, so $\min _{F_{0}} I(g) \leqslant \liminf U_{U_{\delta}} I(g)$. (We write min rather than inf since $\tilde{F}_{0}$ is compact.) However, $\min _{F_{0}} I(g)$ is at least as big as $\inf _{U_{\delta}} I(g)$, since $F_{0} \subset U_{\delta}$. Thus $\min _{F_{0}} I(g)=$ $\lim _{\delta \rightarrow 0} \inf _{U_{\delta}} I(g)$.

\section{Minimizing the rate function on the boundary}

From now on we will work exclusively with graphons rather than with graphs. From theorem 3.1, all questions boil down to 'minimize the rate function over such-and-such region'. The first region we study is the lower boundary of $(e, t)$-space, beginning with the first (flat) scallop. 
Theorem 4.1. If $e \leqslant 1 / 2$ and $t=0$, then $\min _{F_{0}} I(g)=I_{0}(2 e) / 2$, and this minimum is achieved at the graphon

$$
g_{0}(x, y)= \begin{cases}2 e & \text { if } x<\frac{1}{2}<y \text { or } y<\frac{1}{2}<x \\ 0 & \text { otherwise. }\end{cases}
$$

Furthermore, any other minimizer is equivalent to $g_{0}$, corresponding to the same reduced graphon.

Proof. Since $t(g)$ is identically zero, the measure of the set $\left\{(x, y) \in[0,1]^{2} \mid g(x, y)=0\right\}$ is at least $1 / 2$. Otherwise, the graphon $\bar{g}(x, y)=\left\{\begin{array}{ll}1 & \text { if } g(x, y)>0 ; \\ 0 & \text { otherwise, }\end{array}\right.$ would have no triangles and would have edge density greater than $1 / 2$, which is impossible. So we restrict attention to graphons that are zero on a set of measure at least $1 / 2$ and have edge density $e$. From the convexity of $I_{0}$, we know that the graphon minimizing $I$ must be zero on a set of measure $1 / 2$ and must be constant on the rest. Thus $g_{0}$ is a minimizer, and $\min _{F_{0}} I(g)=I\left(g_{0}\right)=I_{0}(2 e) / 2$.

Now suppose that $g$ is another minimizer. Since $g$ is zero on a set of measure $1 / 2$ and is $2 e$ on a set of measure $1 / 2, \bar{g}$ is 1 on a set of measure $1 / 2$, and so describes a graphon with edge density $1 / 2$ and no triangles. This means that $\bar{g}$ describes a complete bipartite graph with the two parts having the same measure. That is, $\bar{g}$ is equivalent to the graphon that equals 1 if $x<\frac{1}{2}<y$ or $y<\frac{1}{2}<x$ and is zero everywhere else. But then $g=2 e \bar{g}$ is equivalent to $g_{0}$.

The situation on the curved scallops is slightly more complicated. Pick an integer $\ell>1$. (The case $\ell=1$ just gives us our first scallop.) If $e \in\left[1-\frac{1}{\ell}, 1-\frac{1}{\ell+1}\right]$, then any graph $G$ with edge density $e$ and the minimum number of triangles has to take the following form (see [PR] for the history). Let

$$
c=\frac{\ell+\sqrt{\ell(\ell-e(\ell+1))}}{\ell(\ell+1)} .
$$

There is a partition of $\{1, \ldots, n\}$ into $\ell$ pieces, the first $\ell-1$ of size $\lfloor c n\rfloor$ and the last of size between $\lfloor c n\rfloor$ and $2\lfloor c n\rfloor$, such that $G$ is the complete $\ell$-partite graph on these pieces, plus a number of additional edges within the last piece. $(\lfloor y\rfloor$ denotes the largest integer greater than or equal to $y$.) These additional edges can take any form, as long as there are no triangles within the last piece.

This means that, after possibly renumbering the vertices, the graphon for such a graph can be written as an uneven $\ell \times \ell$ checkerboard obtained from cutting the unit interval into pieces $V_{k}=[(k-1) c, k c]$ for $k<\ell$ and $V_{\ell}=[(\ell-1) c, 1]$, with the checkerboard being 1 outside the main diagonal, 0 on the main diagonal except the upper right corner, and corresponding to a zero-triangle graph in the upper right corner.

Limits of such graphons in the metric must take the form

$$
g(x, y)= \begin{cases}1 & x<k c<y \text { or } y<k c<x \text { for an integer } k<\ell \\ 0 & (k-1) c<x, y<k c \text { for some integer } k<\ell \\ \text { unspecified } \quad x, y>(\ell-1) c\end{cases}
$$

with

$$
\iiint_{[(\ell-1) c, 1]^{3}} g(x, y) g(y, z) g(z, x) \mathrm{d} x \mathrm{~d} y \mathrm{~d} z=0,
$$

and with $\iint_{[0,1]^{2}} g(x, y) \mathrm{d} x \mathrm{~d} y=e$. Minimizing $I(g)$ on such graphons is easy, since all but the upper right corner of the graphon is fixed. Applying theorem 4.1 to that corner, we get 
Theorem 4.2. If $e>1 / 2$ and $t$ is the smallest value possible, then the minimum of $I(g)$ on $F_{0}$ is achieved by the graphon

$g_{0}(x, y)= \begin{cases}1 & x<k c<y \text { or } y<k c<x \text { for an integer } k<\ell \\ p \quad & (\ell-1) c<x<[1+(\ell-1) c] / 2<y \text { or }(\ell-1) c<y<[1 \\ & +(\ell-1) c] / 2<x \\ 0 \quad \text { otherwise }\end{cases}$

where

$$
p=\frac{4 c(1-\ell c)}{(1-(\ell-1) c)^{2}}
$$

is a number chosen to make $\iint_{[0,1]^{2}} g(x, y) \mathrm{d} x \mathrm{~d} y=e$. Furthermore, any other minimizer is equivalent to $g_{0}$. The minimum value of $I(\mathrm{~g})$ is

$$
I\left(g_{0}\right)=\frac{(1-(\ell-1) c)^{2}}{2} I_{0}(p)
$$

\section{Minimizing near the first scallop}

Now that we know the minimizer at the (lower) boundary, we perturb it to get a minimizer near the boundary.

Theorem 5.1. Pick $e<1 / 2$ and $\epsilon$ sufficiently small. Then the graphon

$$
g(x, y)= \begin{cases}2 e-\epsilon & x<\frac{1}{2}<y \text { or } y<\frac{1}{2}<x \\ \epsilon & \text { otherwise, }\end{cases}
$$

minimizes the rate function to second order in perturbation theory among graphons with $e(g)=e$ and $t(g)=e^{3}-(e-\epsilon)^{3}$. For pointwise small variations $\delta g$ of $g$, the second variation in $I(g)$ is bounded from below by $\frac{1}{2} \iint_{[0,1]^{2}}(\delta g(x, y))^{2} \mathrm{~d} x \mathrm{~d} y$.

Proof. We first consider the first variation in $I(g)$ for general graphons and derive the EulerLagrange equations. It is easy to check that our specific $g$ satisfies these equations. We then consider the second variation in $I(g)$. Note that the function $I_{0}$ satisfies

$$
I_{0}^{\prime}(u)=\frac{1}{2}[\ln (u)-\ln (1-u)], \quad I_{0}^{\prime \prime}(u)=\frac{1}{2}\left[\frac{1}{u}+\frac{1}{1-u}\right] \geqslant 2 .
$$

To find the Euler-Lagrange equations with the constraints that $(e(g), t(g))$ are equal to fixed values $\left(e_{0}, t_{0}\right)$, we use Lagrange multipliers and vary the function $I(g)+\lambda_{1}\left(e(g)-e_{0}\right)+$ $\lambda_{2}\left(t(g)-t_{0}\right)$. To first order, the variation with respect to $g$ is

$$
\begin{aligned}
\delta I(g)= & \int_{0}^{1} \int_{0}^{1} I_{0}^{\prime}(g(x, y)) \delta g(x, y) \mathrm{d} x \mathrm{~d} y+\lambda_{1} \int_{0}^{1} \int_{0}^{1} \delta g(x, y) \mathrm{d} x \mathrm{~d} y \\
& +3 \lambda_{2} \int_{0}^{1} \int_{0}^{1} h(x, y) \delta g(x, y) \mathrm{d} x \mathrm{~d} y,
\end{aligned}
$$

where we have introduced the auxiliary function

$$
h(x, y)=\int_{0}^{1} g(x, z) g(y, z) \mathrm{d} z .
$$

Setting $\delta I(g)$ equal to zero, we get

$$
I_{0}^{\prime}(g(x, y))=-\lambda_{1}-3 \lambda_{2} h(x, y) .
$$


Our particular $g(x, y)$ satisfies this equation with

$$
3 \lambda_{2}=\frac{I_{0}^{\prime}(2 e-\epsilon)-I_{0}^{\prime}(\epsilon)}{2(e-\epsilon)^{2}} .
$$

Next we expand $\delta t$ and $\delta I$ to second order in $\delta g$, ignoring $O\left((\delta g)^{3}\right)$ terms. Since $\delta t=3 \iint h(x, y) \delta g(x, y) \mathrm{d} x \mathrm{~d} y+3 \iiint g(x, y) \delta g(x, z) \delta g(y, z) \mathrm{d} x \mathrm{~d} y \mathrm{~d} z+O\left((\delta g)^{3}\right)$, and since we are holding $e(g)$ and $t(g)$ fixed,

$$
\begin{aligned}
\delta I= & \iint I_{0}^{\prime}(g(x, y)) \delta g(x, y) \mathrm{d} x \mathrm{~d} y+\frac{1}{2} \iint I_{0}^{\prime \prime}(g(x, y))(\delta g(x, y))^{2} \mathrm{~d} x \mathrm{~d} y \\
= & \iint\left(-\lambda_{1}-3 \lambda_{2} h(x, y)\right) \delta g(x, y) \mathrm{d} x \mathrm{~d} y+\frac{1}{2} \iint I_{0}^{\prime \prime}(g(x, y))(\delta g(x, y))^{2} \mathrm{~d} x \mathrm{~d} y \\
= & -\lambda_{1} \delta e-\lambda_{2} \delta t+3 \lambda_{2} \iiint g(x, y) \delta g(x, z) \delta g(y, z) \mathrm{d} x \mathrm{~d} y \mathrm{~d} z \\
& \quad+\frac{1}{2} \iint I_{0}^{\prime \prime}(g(x, y))(\delta g(x, y))^{2} \mathrm{~d} x \mathrm{~d} y \\
= & 3 \lambda_{2} \iiint g(x, y) \delta g(x, z) \delta g(y, z) \mathrm{d} x \mathrm{~d} y \mathrm{~d} z+\frac{1}{4} \iint I_{0}^{\prime \prime}(g(x, y)) \delta g(x, y)^{2} \mathrm{~d} x \mathrm{~d} y \\
& \quad+\frac{1}{4} \iint I_{0}^{\prime \prime}(g(x, y)) \delta g(x, y)^{2} \mathrm{~d} x \mathrm{~d} y .
\end{aligned}
$$

We have split the $\iint I^{\prime \prime} \delta g^{2}$ term into two pieces, as we will be applying different estimates to each piece.

Since $h(x, y)$ and $I^{\prime \prime}(g)$ are piecewise constant, all of our integrals break down into integrals over different quadrants. Let $R_{1}$ and $R_{2}$ be the following subsets of $[0,1]^{2}$ :

$R_{1}=\{x, y<1 / 2\} \cup\{x, y>1 / 2\}, \quad R_{2}=\{x<1 / 2<y\} \cup\{y<1 / 2<x\}$.

For each $z$, we define the functions $f_{1}(z)=\int_{0}^{1 / 2} \delta g(x, z) \mathrm{d} x$ and $f_{2}(z)=\int_{1 / 2}^{1} \delta g(x, z) \mathrm{d} x$. The second variation in $I$ is then

$$
\begin{aligned}
& \frac{1}{4} \iint_{[0,1]^{2}} I_{0}^{\prime \prime}(g) \\
& \quad \delta g(x, y)^{2} \mathrm{~d} x \mathrm{~d} y+\frac{I_{0}^{\prime \prime}(\epsilon)}{4} \iint_{R_{1}} \delta g(x, y)^{2} \mathrm{~d} x \mathrm{~d} y \\
& +\frac{I_{0}^{\prime \prime}(2 e-\epsilon)}{4} \iint_{R_{2}} \delta g(x, y)^{2} \mathrm{~d} x \mathrm{~d} y+3 \lambda_{2} \int_{0}^{1} \mathrm{~d} z\left[\epsilon \iint_{R_{1}} \delta g(x, z) \delta g(y, z) \mathrm{d} x \mathrm{~d} y\right. \\
& \left.+(2 e-\epsilon) \iint_{R_{2}} \delta g(x, z) \delta g(y, z) \mathrm{d} x \mathrm{~d} y\right] \\
& =\frac{1}{4} \iint_{[0,1]^{2}} I_{0}^{\prime \prime}(g(x, y)) \delta g(x, y)^{2} \mathrm{~d} x \mathrm{~d} y+\frac{I_{0}^{\prime \prime}(\epsilon)}{4} \iint_{R_{1}} \delta g(x, z)^{2} \mathrm{~d} x \mathrm{~d} z \\
& \left.+\frac{I_{0}^{\prime \prime}(2 e-\epsilon)}{4} \iint_{R_{2}} \delta g(x, z)^{2} \mathrm{~d} x \mathrm{~d} z+3 \lambda_{2} \int_{0}^{1} \epsilon\left[f_{1}(z)^{2}+f_{2}(z)^{2}\right)\right] \\
& +2(2 e-\epsilon) f_{1}(z) f_{2}(z) \mathrm{d} z .
\end{aligned}
$$

Note that by Cauchy-Schwarz,

$$
\begin{aligned}
& \int_{0}^{1 / 2}(\delta g(x, z))^{2} \mathrm{~d} x \geqslant 2\left(\int_{0}^{1 / 2} \delta g(x, z) \mathrm{d} x\right)^{2}=2 f_{1}(z)^{2} \\
& \int_{1 / 2}^{1}(\delta g(x, z))^{2} \mathrm{~d} x \geqslant 2\left(\int_{1 / 2}^{1} \delta g(x, z) \mathrm{d} x\right)^{2}=2 f_{2}(z)^{2}
\end{aligned}
$$


Since $I_{0}^{\prime \prime}(\epsilon)$ and $I_{0}^{\prime \prime}(2 e-\epsilon)$ are positive, $\delta I$ is bounded from below by

$$
\begin{aligned}
\frac{1}{4} \iint_{[0,1]^{2}} I_{0}^{\prime \prime}( & g(x, y)) \delta g(x, y)^{2} \mathrm{~d} x \mathrm{~d} y+\frac{I^{\prime \prime}(\epsilon)}{2}\left[\int_{0}^{1 / 2} f_{1}(z)^{2} \mathrm{~d} z+\int_{1 / 2}^{1} f_{2}(z)^{2} \mathrm{~d} z\right] \\
+ & \frac{I_{0}^{\prime \prime}(2 e-\epsilon)}{2}\left[\int_{0}^{1 / 2} f_{2}(z)^{2} \mathrm{~d} z+\int_{1 / 2}^{1} f_{1}(z)^{2} \mathrm{~d} z\right] \\
+ & 3 \lambda_{2} \int_{0}^{1} \mathrm{~d} z\left[\epsilon\left(f_{1}(z)^{2}+f_{2}(z)^{2}\right)+2(2 e-\epsilon) f_{1}(z) f_{2}(z)\right] .
\end{aligned}
$$

Collecting terms and applying equation (26), this bound becomes

$$
\begin{gathered}
\frac{1}{4} \iint_{[0,1]^{2}} I_{0}^{\prime \prime}(g(x, y)) \delta g(x, y)^{2} \mathrm{~d} x \mathrm{~d} y+\int_{0}^{1 / 2} \mathrm{~d} z\left[c_{1} f_{1}(z)^{2}+c_{2} f_{2}(z)^{2}+2 c_{3} f_{1}(z) f_{2}(z)\right] \\
+\int_{1 / 2}^{1} \mathrm{~d} z\left[c_{1} f_{2}(z)^{2}+c_{2} f_{1}(z)^{2}+2 c_{3} f_{1}(z) f_{2}(z)\right]
\end{gathered}
$$

where

$$
\begin{aligned}
& c_{1}=\frac{I_{0}^{\prime \prime}(\epsilon)}{2}+\frac{\epsilon\left(I_{0}^{\prime}(2 e-\epsilon)-I_{0}^{\prime}(\epsilon)\right)}{2(e-\epsilon)^{2}} \\
& c_{2}=\frac{I_{0}^{\prime \prime}(2 e-\epsilon)}{2}+\frac{\epsilon\left(I_{0}^{\prime}(2 e-\epsilon)-I_{0}^{\prime}(\epsilon)\right)}{2(e-\epsilon)^{2}} \\
& c_{3}=\frac{(2 e-\epsilon)\left(I_{0}^{\prime}(2 e-\epsilon)-I_{0}^{\prime}(\epsilon)\right)}{2(e-\epsilon)^{2}} .
\end{aligned}
$$

Note that all coefficients are positive, and that $c_{2}>1$. As $\epsilon \rightarrow 0, c_{1}$ goes to $+\infty$ as $1 / \epsilon$, while $c_{3}$ only diverges as $-\ln (\epsilon)$. Since $c_{1} c_{2}>c_{3}^{2}$ for small $\epsilon$, the integrand for each $z$ is positive semi-definite, so the integral over $z$ is non-negative, and we obtain

$$
\delta I \geqslant \frac{1}{4} \iint I_{0}^{\prime \prime}(g) \delta g^{2} \geqslant \frac{1}{2} \iint \delta g(x, y)^{2},
$$

where we used the fact that $I_{0}^{\prime \prime}(u) \geqslant 2$ for all $u$.

Any global minimizer must be $O(\epsilon)$ close to $g_{0}$, and hence $O(\epsilon)$ close to our specified perturbative minimizer. This means that the only way for them to differ is through a complicated bifurcation of minimizers at $g_{0}$, despite the uniform bounds on $\delta I$ as we approach the boundary. The difference between these hypothetical minimizers and $g_{0}$ would not be pointwise small, but would merely be small in an $L^{1}$ sense.

For instance, consider graphons of the form

$$
g(x, y)= \begin{cases}p & x<c<y \text { or } y<c<x \\ \alpha & x, y<c \\ \beta & x, y>c\end{cases}
$$

where $c$ is a parameter that we will vary and $p, \alpha$ and $\beta$ are constants that depend on $c$. For each $c$ sufficiently close to $1 / 2$, it is possible to find a graphon of this form such that $\iiint g(x, y) g(y, z) g(x, z) \mathrm{d} x \mathrm{~d} y \mathrm{~d} z=t$ and $\iint g(x, y) \mathrm{d} x \mathrm{~d} y=e$, and such that the EulerLagrange equations (25) are satisfied. Call this graphon $g_{c}(x, y)$. A lengthly calculation shows that

$$
\left.\frac{\partial^{2} I\left(g_{c}\right)}{\partial c^{2}}\right|_{c=1 / 2} \geqslant 16 e^{2}
$$

for small $t$, indicating that (nearly) bipartite graphs with pieces of unequal size have a higher rate function than $g$. This provides strong evidence that our perturbative solution is in fact a global minimizer for sufficiently small $t$. 
Corollary 5.2. Assuming our perturbative solution is the global optimizer, there is a phase transition near the boundary point $(1 / 2,0)$ between the first and second scallop.

Proof. Our perturbative solution yields a formula for the entropy:

$$
s_{e, t}=-\frac{1}{2}\left[I_{0}(\epsilon)+I_{0}(2 e-\epsilon)\right] .
$$

This formula for the entropy cannot be extended analytically beyond $e=(1+\epsilon) / 2$, as $\partial^{2} s / \partial e^{2}$ diverges as $e \rightarrow(1+\epsilon) / 2$. However, $e=(1+\epsilon) / 2$ corresponds to $t=\left(\epsilon^{3}+3 \epsilon\right) / 4$, or, using the more basic variable $e$,

$$
t=\left[(2 e-1)^{3}+3(2 e-1)\right] / 4,
$$

which is in the interior of $(e, t)$ space. (Since the graphon $g(x, y)$ is nowhere zero, it differs in form from the graphons describing graphs with minimal $t$.) Thus $s_{e, t}$ must fail to be analytic in some neighborhood of the first scallop.

Of course there must also be a phase transition, presumably from this bipartite phase to a homogeneous phase, if one fixes $e$ and raises $t$, which we see as follows.

Corollary 5.3. Assuming our perturbative solution is the global optimizer, there is a phase transition as one raises $t$, for any fixed $0<e<1 / 2$.

Proof. Recall from corollary 5.2 the connection between $t$ and $\epsilon$ :

$$
t=\epsilon^{3}-(e-\epsilon)^{3} .
$$

Note that $t$ is an increasing function of $\epsilon$ and reaches the value $2 e^{3}$ when $\epsilon=2 e$. From equation (41) for the entropy we see that it cannot be extended analytically to $t>2 e^{3}$, yet for $e<1 / 2$ we have $2 e^{3}<e^{2}<e^{3 / 2}$ so $(e, t)$ is in the interior of the phase space (Figure 1).

\section{Conclusion}

Our goal was to analyze possible phase transitions between multipartite phases of complex networks, analogous to solid/solid transitions in materials. To this end we adapted the Strauss model [St], defined in the grand canonical ensemble, to a microcanonical ensemble. It is appropriate at this point to review the 'equivalence of ensembles' in statistical physics.

In thermodynamics the concavity of the entropy $S(E, N, V)$, as a function of internal energy $E$, particle number (or mass) $N$ and volume $V$, and the interpretation of equilibrium states as states maximizing the entropy, are both fundamental; see for instance [Ca, Ma]. Lagrange multipliers can be used to convert this optimization criterion of the entropy to an equivalent optimization criterion of the free energy, where the free energy is the Legendre transform of the entropy [Do, Ca, Ma]. It is important that the Legendre transform between the entropy and free energy be invertible so the two optimization schemes are equivalent, and this follows from the concavity of the entropy. (See section 26 of [Ro] for the mathematics of the Legendre transform between convex functions.)

Statistical mechanics supplies a model for thermodynamic states, as probability distributions on mechanical multiparticle states. From a given short range particle interaction one can then (in principle) compute the internal energy $E$ and entropy $S$, and prove the above two features of the entropy: its concavity and its optimization role for equilibrium states. To do this one uses the basic Boltzmann/Gibbs ansatz: that the entropy $S(E, N, V)$ is proportional to

$$
-\sum_{j} \rho_{j} \ln \left(\rho_{j}\right)
$$




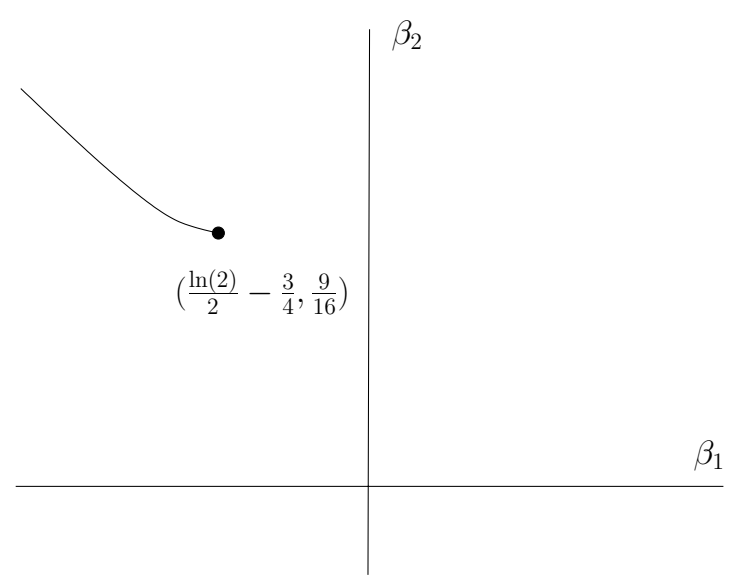

Figure 2. The curve of all singularities of $\psi_{\beta_{1}, \beta_{2}}$ for $\beta_{2}>-1 / 2$.

where $\rho_{j}$ is the probability of multiparticle state $j$, and the equilibrium state is that probability distribution $\left\{\rho_{j}\right\}$ on the set $\Xi(E, N, V)$, of multiparticle states of energy $E$ and particle number $N$ in volume $V$, which maximizes the entropy [R1, R2, Ge, Wi, Ma]. (Note that in taking an infinite volume limit, which we must do to obtain equivalence of ensembles, one can divide the entropy's variables by volume, and consider the entropy density as a function of particle and energy density.)

The equivalence of ensembles in statistical mechanics is basically a strengthening of the equivalence in thermodynamics between entropy $s_{e, t}$ and free energy $\psi_{\beta_{1}, \beta_{2}}$, corresponding to Lagrange multipliers $\beta_{1}$ and $\beta_{2}$, which follows from the concavity of the entropy. With the modeling of the thermodynamic states this now implies a bijection $(e, t) \longleftrightarrow\left(\beta_{1}, \beta_{2}\right)$ such that $s_{e, t}$ and $\psi_{\beta_{1}, \beta_{2}}$ have the same optimizing states, at least off some manageable sets of parameter pairs corresponding to 'phase coexistence' where the bijection can degenerate to a many-to-one map [Ge].

In exponential random graph models, which are mean field rather than short range, the entropy need not be concave [TET] and indeed this fails in an obvious way for the specific model we are analyzing, the Strauss model, since even the domain $R$ of the entropy is not convex (see figure 1). Therefore in the infinite node limit of the model the free energy density $\psi_{\beta_{1}, \beta_{2}}$, need not be equivalent to the entropy density $s_{e, t} ; \psi_{\beta_{1}, \beta_{2}}$ can be obtained from $s_{e, t}$ by Legendre transform, but it may not be possible to recover $s_{e, t}$ from $\psi_{\beta_{1}, \beta_{2}}$. Inequivalence can result from the existence of graphons maximizing $s_{e, t}$ for some $(e, t)$ which are not maximizers of $\psi_{\beta_{1}, \beta_{2}}$ for any $\left(\beta_{1}, \beta_{2}\right)$. Specific instances of such loss of information in $\psi_{\beta_{1}, \beta_{2}}$ are shown in a future paper [RS], but one consequence can already be seen in the transitions, studied previously [PN, CD, RY] in the grand canonical ensemble, between independent-edge graphs across a phase transition curve in the phase space; see figure 2 for the Strauss model. Such 'free particle' graphs, with only edge density $e$ as a variable, optimize $\psi_{\beta_{1}, \beta_{2}}$ for $\left(\beta_{1}, \beta_{2}\right)$ throughout the upper half of the grand canonical phase space, so $e$ is a function of $\left(\beta_{1}, \beta_{2}\right)$ off the transition curve there; see [RY] for details. These graphs all lie on the curve $t=e^{3}$ in figure 1 , not a two-dimensional region in that microcanonical phase space, making it difficult to use singularities of the free energy $\psi_{\beta_{1}, \beta_{2}}$ to imply singularities of the entropy $s_{e, t}$. For this reason we have focused here on phase transitions in the lower region of the microcanonical phase space, figure 1. 
We have shown that our (2-parameter, bipartite) graphons $g$ of theorem 5.1 maximize the entropy density at least to second order in perturbation theory, among graphons with a limited range of edge and triangle densities. Assuming the $g$ are actually global maximizers we then proved the entropy density would have to lose analyticity as the edge density of the graphon approaches the tripartite regime. We also show that the entropy density must suffer a phase transition as the triangle density is raised sufficiently high, presumably from the structured bipartite phase to a homogeneous phase of higher triangle density.

We expect that a more complicated analysis could produce appropriate graphons $g^{(k)}, k \geqslant 1$, near each of the higher edge density (multipartite) graphons of minimal triangle density, with a transition near each scallop intersection. Intuitively this suggests a mechanism whereby as edge density is increased, near the minimum triangle density graphon, the system progressively transitions through finer and finer structure; for high edge density most graphs would consist of many interacting 'parts'.

Our results on phase transitions require that the graphons of theorem 5.1 be in fact global, not just local, maximizers of the entropy density. In a future paper [RS] we use a symmetry to prove that these graphons are indeed the unique global maximizers at least for triangle density in the range $0 \leqslant t \leqslant 1 / 8$ and edge density $e=1 / 2$, and we can then see a transition on this curve. However we still cannot prove the graphons are the global optimizers of entropy density for $(e, t)$ in any two-dimensional region, as is needed to fully justify the notion of a structured phase. (See $[A R]$ for a variant of this approach.)

In conclusion we emphasize that our key tool was theorem 3.1, an optimization formula for the asymptotic entropy density, and made essential use of the graph limit formalism. The graphon formalism is a powerful tool for dealing with the infinite size limit in mean field models, and we have used it to make some progress on understanding the structure of asymptotically large graphs near the extreme of low triangle density.

\section{Acknowledgments}

We gratefully acknowledge useful discussions with Francesco Maggi and Peter Winkler. This work was partially supported by NSF grants DMS-1208941 and DMS-1101326.

\section{References}

[AR] Aristoff D and Radin C 2013 Emergent structures in large networks J. Appl. Probab. at press (arXiv:1110.1912)

[BCL] Borgs C, Chayes J and Lovász L 2010 Moments of two-variable functions and the uniqueness of graph limits Geom. Funct. Anal. 19 1597-619

[BCLSV] Borgs C, Chayes J, Lovász L, Sós V T and Vesztergombi K 2008 Convergent graph sequences I: subgraph frequencies, metric properties, and testing Adv. Math. 219 1801-51

[Ca] Callen H B 1960 Thermodynamics (New York: Wiley)

[CD] Chatterjee S and Diaconis P 2011 Estimating and understanding exponential random graph models arXiv:1102.2650v3

[CV] Chatterjee S and Varadhan S R S 2011 The large deviation principle for the Erdôs-Rényi random graph Eur. J. Comb. 32 1000-17

[Do] Dorlas T C 1999 Statistical Mechanics: Fundamentals and Model Solutions (London: Institute of Physics Publishing)

[Ge] Georgii H-O 1995 The equivalence of ensembles for classical systems of particles J. Stat. Phys. 80 1341-78

[Lov] Lovász L 2012 Large Networks and Graph Limits (Providence, RI: American Mathematical Society)

[LS1] Lovász L and Szegedy B 2006 Limits of dense graph sequences J. Combin. Theory Ser. B 96 933-57

[LS2] Lovász L and Szegedy B 2007 Szemerédi’s lemma for the analyst Geo. Funct. Anal. 17 252-70

[LS3] Lovász L and Szegedy B 2011 Finitely forcible graphons J. Combin. Theory Ser. B 101 269-301

[Ma] Ma S-K 1985 Statistical Mechanics (Singapore: World Scientific) 
[Ne] Newman M E J 2010 Networks: an Introduction (Oxford: Oxford University Press)

[PN] Park J and Newman M E J 2005 Solution for the properties of a clustered network Phys. Rev. E 72026136

[PR] Pikhurko O and Razborov A 2012 Asymptotic structure of graphs with the minimum number of triangles arXiv: 1203.4393

[R1] Ruelle D 1969 Statistical Mechanics; Rigorous Results (New York: Benjamin)

[R2] Ruelle D 1978 Thermodynamic Formalism (New York: Addison-Wesley)

[Ro] Rockafellar R T 1970 Convex Analysis (Princeton, NJ: Princeton University Press)

[RS] Radin C and Sadun L 2013 Singularities in the entropy of asymptotically large simple graphs arXiv:1302:3531

[RY] Radin C and Yin M 2013 Phase transitions in exponential random graphs Ann. Appl. Probab. at press (arXiv:1108.0649)

[St] Strauss D 1986 On a general class of models for interaction SIAM Rev. 28 513-27

[TET] Touchette H, Ellis R S and Turkington B 2004 Physica A 340 138-46

[Wi] Wightman A S 1979 Convexity and the notion of equilibrium state in thermodynamics and statistical mechanics Convexity in the Theory of Lattice Gases ed R Israel (Princeton, NJ: Princeton University Press) pp 9-75 